\title{
Consent and confidentiality in clinical genetic practice: guidance on genetic testing and sharing genetic information
}

\author{
Anneke Lucassen and Alison Hall
}

The use of genetic tests in healthcare is rapidly increasing. This is in part because rapid advances in technology make such testing quicker and cheaper, but also because there is greater evidence to guide treatments or preventative options as a result of testing.

Historically, genetic tests in the UK have been performed largely by regional genetics services - a series of NHS clinics to which individuals, or families, can be referred. Assessment of a family history and/or signs and symptoms may provide a diagnosis, indicate which genes should be tested, or allow advice on risks of disease. This model works well for highly penetrant genetic conditions and allows coordination of the varied surveillance regimens or interventions required as well as providing the opportunity for communication about the complexities of genetic information.

Although such high risk conditions are relatively rare, almost any disease will have some genetic component and recent rapid technological advances mean that more and more can be identified at costs affordable to the NHS. While achieving genetic diagnoses in the past could be likened to the proverbial search for the needle in a haystack and, once found, at best offered only reproductive choices, this is rapidly changing. Genetic (or genomic) testing is beginning to impact on most areas of medicine. Calls have been made to prepare for a rapid transformation in practice so that genetic, or genomic, ${ }^{a}$ tests are integrated into mainstream medical care. ${ }^{1}$ The expertise gained by genetic services in dealing with communication and familial aspects will be integral to this transformation. ${ }^{2}$

Although some health professionals may view a genetic test as simply another laboratory request form to be completed, there are complexities to consider. Firstly, the language and concepts surrounding genetic information and associated notions of identity or stigma can be more complex than those surrounding, say, a haemoglobin check, and this needs to be incorporated in the consent process of certain tests. Secondly, the results of a genetic test in one person may reveal information about the likelihood of disease in family members, so that consent for relevant communication, and the limits of confidentiality, may need to be considered.

As a consequence, the Joint Committee on Medical Genetics, a tripartite committee of the Royal Colleges of Physicians, Pathologists and British Society of Human Genetics, has

Anneke Lucassen, professor/consultant in clinical genetics, University of Southampton; Alison Hall, senior policy adviser, PHG Foundation recently updated its guidance on consent and confidentiality in clinical genetic practice. ${ }^{3}$ The guidance explores the ethical and legal challenges inherent in managing such sensitive, personal, yet at the same time often familial, information. By using a series of worked illustrative examples based upon cases that arose in clinical practice (some examples are given in Boxes 1-3), the report aims to guide healthcare professionals through the complex web of legislation and professional guidance relating to the use of genetic data and samples. It recognises the growing uncertainty among healthcare professionals about the duties and responsibilities they may have towards relatives of a patient, including when it might be appropriate or legitimate to utilise genetic information from one person for the benefit of another in order to recommend appropriate interventions. At the same time it attempts to formalise procedures to recognise that many service users attend genetic clinics not just for themselves, but often (at least in part) in order to help their families.

There needs to be an appropriate balance between respect for individual consent and confidentiality and the appropriate sharing of relevant information with others at risk. That is to say, the public interest in maintaining a confidential genetic service needs to be balanced against the public interest in the prevention of harm. In this context the harm might be to individuals not knowing about their genetic risk, and thus not being able to avail themselves of particular treatments or preventative options. Recent General Medical Council guidance on confidentiality ${ }^{4}$ has included some genetic examples providing helpful support for genetic medicine, although these largely address the situation where one person actively witholds information that might benefit others. In practice, this is rare, and it is much more common that relevant communication is incomplete, or that relatives do not know what, or how, to communicate, or that professionals are simply uncertain about what consent has been given by others.

The guidance recommends a more proactive approach to information sharing in which:

- The approach to obtaining consent is robust and transparent, so that patients are informed in general terms about how their

\footnotetext{
a 'Genetic' refers to genes, 'genomic' to genomes. The word 'genome' is a conflation of the words gene and chromosome and was first coined in the 1990s to indicate the study of genes and their surrounding DNA. While the terms 'genetic medicine' and 'genomic medicine' have been variably defined by experts, in this article the terms are viewed as largely overlapping.
} 
sample (and the information derived from it) might be used. Apart from use for relatives, this might also include using remnants of the sample for quality assurance or incorporating test results into national data sets. Consent may also need to incorporate the wider scope of testing inherent in newer technologies; the fact that these might uncover unexpected or incidental information (either about biological relationships or about risks of disease), and that knowledge about risks and interventions is likely to evolve rapidly over the next few years. Model consent forms that address the particular nuances of genetic practice are provided.

- Family history and clinical information are shared between relevant health professionals if appropriate to the healthcare needs of family members.

Box 1. Communication within families; appropriate alerting of relatives at risk; anonymisation of individual results.

Bob has a genetic test for a cardiac rhythm problem that runs in his family and causes sudden death. The test shows he has a faulty gene and after consultation with the cardiologists he has a cardiac defibrillator implanted under his skin. He has several siblings, who each have a one in two chance of having inherited the same cardiac risk. Should the onus be on Bob to tell them to seek medical care? Does letting relatives know depend on the effectiveness of interventions available? How can communicating this information be managed sensitively enough to respect the potential desire of relatives not to know? Is the information confidential to Bob, because it was discovered in him, or is it in fact confidential to his biological family, because they may also be bearers of the faulty gene?

Box 2. Clinically relevant incidental findings from genomic tests; appropriate routes of communication; planning future management.

Amy has taken her five-year-old daughter to be investigated for learning difficulties. Routine investigations show a deletion of genetic material which provides a diagnosis, but also predicts a high chance of bowel cancer (adenomatous polyposis) in her 20s and 30s. Further investigations reveal that she has inherited this from her father. He too is now known to be at high risk of bowel cancer, and regular surveillance as well as probable surgery is indicated, yet he lives in another part of the country and had only provided a blood sample to help his daughter. He now receives the information via his estranged wife and is unhappy about receiving unsolicited information.

Box 3. Legislation designed to preserve confidences and risk assessment in relatives.

George has a strong family history of cancer. In order to determine whether there is a strong inherited component to this, tests are requested on the stored tumour blocks of deceased relatives. The request has been refused by laboratory staff since it is thought that none of the relatives consented to testing 'for the benefit of a relative' during life. The Human Tissue Act (2004) is given as justification for this refusal. Without these tests, a genetic test in George cannot exclude that he is at high risk.
- Certain risks of disease and/or genetic diagnoses are communicated to relevant family members (via an agreed route such as the general practitioner, genetic services or the patient). Such communication may be given greater emphasis where there is potential to modify disease risks through medical intervention.

- Relevant information is shared in an anonymised fashion where possible, so that relatives can be appropriately alerted, without disclosing sensitive information about others.

As well as detailed analysis of consent and confidentiality practices relating to genetics, the guidance provides an applied analysis of relevant legislation, in particular the Human Tissue Act (HTA, 2004), the Data Protection Act (DPA, 1998) and the Mental Capacity Act (MCA, 2005). All three laws were predicated upon individually focused medical practice at their core so that applying them to familial medicine is not always straightforward. For example, how should the requirements of the DPA be interpreted when taking and recording a detailed family history? How does the HTA permit analysis of samples for the benefit of relatives? Detailed flowcharts summarise practical sequential steps, while worked clinical examples flesh out the types of problems encountered in practice and provide pragmatic solutions, or means to solve particular dilemmas. The UK forum for discussion of particular ethical issues in genetic medicine can provide ongoing support ${ }^{5}$ and engaging with local clinical ethics committees can also be helpful.

It is hoped that this guidance provides a constructive framework for healthcare professionals to reflect on their own practice, and thus to facilitate integration of familial aspects of genetic medicine into appropriate and legitimate clinical practice. More generally, it is hoped that it will also facilitate ongoing discussions about genetic and genomic medicine and their integration into mainstream medicine.

\section{References}

1 House of Lords report on genomic medicine, www.publications. parliament.uk/pa/ld200809/ldselect/ldsctech/107/107i.pdf

2 Genetics Education Centre, www.geneticseducation.nhs.uk/

3 Royal College of Physicians, Royal College of Pathologists and British Society for Human Genetics. Consent and confidentiality in clinical genetic practice: guidance on genetic testing and sharing genetic information, 2nd edn. Report of the Joint Committee on Medical Genetics. London: RCP, RCPath, 2011. www.rcplondon.ac.uk/sites/default/files/ consent_and_confidentiality_2011_0.pdf

4 www.gmc-uk.org/static/documents/content/Confidentiality_0910.pdf

5 UK Genethics forum, www.genethicsclub.org

Address for correspondence: Professor A Lucassen, University of Southampton, Faculty of Medicine and Wessex Clinical Genetics Service, Mailpoint 105, Princess Anne Hospital, Coxford Road, Southampton S016 5YA.

Email: annekel@soton.ac.uk 